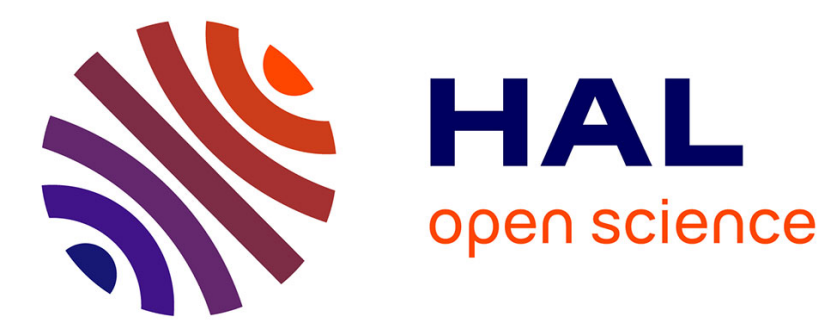

\title{
Actuator fault estimation observer design for discrete-time linear parameter-varying descriptor systems
}

Zhenhua Wang, Mickael Rodrigues, Didier Theilliol, Yi Shen

\section{To cite this version:}

Zhenhua Wang, Mickael Rodrigues, Didier Theilliol, Yi Shen. Actuator fault estimation observer design for discrete-time linear parameter-varying descriptor systems. International Journal of Adaptive Control and Signal Processing, 2015, 29 (2), pp.242-258. 10.1002/acs.2469 . hal-00936400

\section{HAL Id: hal-00936400 https://hal.science/hal-00936400}

Submitted on 25 Jan 2014

HAL is a multi-disciplinary open access archive for the deposit and dissemination of scientific research documents, whether they are published or not. The documents may come from teaching and research institutions in France or abroad, or from public or private research centers.
L'archive ouverte pluridisciplinaire $\mathbf{H A L}$, est destinée au dépôt et à la diffusion de documents scientifiques de niveau recherche, publiés ou non, émanant des établissements d'enseignement et de recherche français ou étrangers, des laboratoires publics ou privés. 


\title{
Actuator fault estimation observer design for discrete-time linear parameter-varying descriptor systems
}

\author{
Zhenhua Wang $^{1}$, Mickael Rodrigues ${ }^{2, *, \dagger}$, Didier Theilliol ${ }^{3}$, Yi Shen ${ }^{1}$ \\ ${ }^{1}$ School of Astronautics, Harbin Institute of Technology, 150001, Harbin, P.R. China \\ ${ }^{2}$ Automatic and Process Control Laboratory, University of Lyon, Lyon, F-69003, France; Université Lyon 1,CNRS \\ UMR 5007, Villeurbanne, F-69622, France \\ ${ }^{3}$ Centre de Recherche en Automatique de Nancy, Université de Lorraine, CNRS UMR 7039, F-54506 \\ Vandoeuvre-les-Nancy, France
}

\section{SUMMARY}

This paper deals with actuator fault estimation for a class of discrete-time Linear Parameter-Varying (LPV) descriptor systems. By considering the fault as an auxiliary state vector, an augmented system is established. Then, a fault estimation observer is designed based on the augmented system. In this paper, the fault estimation observer design is formulated as a Linear Matrix Inequality (LMI) feasibility problem. Therefore, all parameters of the observer can be simultaneously designed by solving a set of strict LMIs. In order to attenuate the effect of the unknown disturbance, fault variation, and measurement noise, we further propose a robust fault estimation observer design method, which is the main contribution of this paper. Finally, performance of the proposed robust fault estimation observer is shown through the application to a trucktrailer model. Copyright (c) 2013 John Wiley \& Sons, Ltd.

Received ...

KEY WORDS: Fault estimation; discrete-time; Linear Parameter-Varying (LPV); descriptor systems; observer design; Linear Matrix Inequality (LMI)

\footnotetext{
*Correspondence to: Mickael Rodrigues, Automatic and Process Control Laboratory, University of Lyon, France.

†mickael.rodrigues@univ-lyon1.fr 


\section{INTRODUCTION}

It is known that faults may lead to performance degradation or even serious system damages. As a result, it is essential to detect and identify faults so that necessary protective actions can be taken in advance. Therefore, fault diagnosis techniques have received considerable attention. During the past decades, model-based fault diagnosis has been most widely considered and various methods have been proposed in the literature, see e.g. [1-4] and the references therein.

In the literature, most of the existing fault diagnosis methods are based on Linear Time-Invariant (LTI) systems. Although much attention has been devoted to fault diagnosis for nonlinear systems $[5,6]$, extension of the fault diagnosis approaches in linear systems to general nonlinear case is still a challenging problem. Since Linear Parameter-Varying (LPV) model can be used to approximate a large class of nonlinear systems, the LPV representation has often been used for modeling and control of nonlinear systems [7, 8]. In recent years, a few scholars have proposed several fault diagnosis methods for LPV systems [9-11]. For instance, [9] proposed a fault reconstruction method for a class of LPV systems using LPV sliding mode observer. In [10], the authors proposed a fault estimation approach for discrete-time LPV systems based on robust dynamic inversion technique. [11] developed a sensor fault isolation and estimation method for nonlinear systems described by polytopic LPV representation. However, it should be noted that these papers only deal with regular systems.

On the other hand, the descriptor systems (also known as singular systems, generalized systems, and implicit systems) appear in many fields, e.g. power systems, electrical networks, and mechanical systems $[12,13]$. In the past decades, many results have been reported on analysis and design of descriptor systems, see e.g. $[12,13]$ and the references therein. However, there are limited papers on fault diagnosis methods for descriptor systems [14-20]. The authors in [14] proposed a fault estimation method for continuous-time nonlinear descriptor systems by using on-line learning methodology. [15] used generalized unknown input observer to deal with the robust fault detection problem for linear descriptor systems. In [16], unknown input observer was used to generate residuals to detect and isolate faults for the descriptor systems and the fault reconstruction was 
achieved by sliding mode observer. More recently, the authors in [17] investigated fault detection for discrete-time switched descriptor systems with time-varying state delays. However, to the best knowledge of the authors, fault diagnosis for LPV descriptor systems has not been well investigated yet [18-20]. [18] designed a robust fault detection filter for a class of nonlinear descriptor systems described by LPV form with globally Lipschitz term. The main idea of [18] is to formulate the robust fault detection filter design as an $H_{-} / H_{\infty}$ problem. However, the methods in [18] only address the fault detection problem but the fault magnitudes cannot be estimated. As pointed out in [21], accurate and timely fault estimation/identification is an important antecedent for satisfactory control reconfiguration. Therefore, it is important to develop fault estimation methods for descriptor systems. In [19], the authors proposed a fault detection, isolation and estimation scheme via unknown input Proportional Integral (PI) observers. It should be noticed that [19] deals with continuous LPV descriptor systems while this paper focuses on the discrete-time case. Moreover, our paper considers how to attenuate the influence of the measurement noise, which is not considered in [19]. In view of this point, the proposed method is more practical than that from [19]. In [20], an actuator fault estimation method for discrete-time LPV descriptor systems is proposed by using PI observer. However, the fault distribution matrix in [20] is constant, which restricts its scope of application. Moreover, external disturbance is not considered in [20]. In view of this, we present this paper to improve the method in [20]. Herein, it is interesting to mention this paper is different from the work in [22]. In [22], discrete-time Takagi-Sugeno systems with sensor faults are first formulated as a descriptor representation, and then an $H_{-} / H_{\infty}$ fault detection filter is designed based on the obtained descriptor system. In other words, [22] deals with fault detection for regular systems by using the technique of descriptor systems. However, this paper studies discrete-time LPV descriptor systems with faults. It is known that descriptor system representation is a generalization of the regular system. Therefore, the proposed method is more general than that of [22].

The aim of this paper is to develop a fault estimation method for a class of discrete-time LPV descriptor systems. In this paper, the constant fault distribution matrix considered in [20] is relaxed to a parameter-varying matrix. As a result, the proposed method has a broader application scope. 
Moreover, we also consider unknown disturbance, fault variation, and measurement noise in this paper. The basic idea of this paper is to construct an augmented system by taking the fault as an auxiliary state vector, and then design a fault estimation observer based on this augmented system. The main contribution of the proposed method lies in the following aspects. First, a new fault estimation observer with a novel structure is proposed for discrete-time LPV descriptor systems. The proposed fault estimation observer can be designed by solving a set of Linear Matrix Inequalities (LMIs). Second, a robust observer design method is proposed to attenuate the effect of unknown disturbance, fault variation, and measurement noise on fault estimation.

The rest of this paper is organized as follows. Section 2 briefly introduces the problem to be studied. In Section 3, a new fault estimation observer for discrete-time LPV descriptor systems is proposed, and the observer design is formulated as a LMI feasibility problem. In order to deal with unknown disturbance, fault variation, and measurement noise, Section 4 proposes a robust fault estimation observer design method. In Section 5, a truck-trailer example is used to show the effectiveness and performance of the proposed methods. Finally, conclusions are given in Section 6.

\section{PROBLEM STATEMENT}

Consider the following class of discrete-time LPV descriptor system

$$
\left\{\begin{array}{l}
E x(k+1)=\sum_{i=1}^{h} \rho_{i}(\theta(k))\left(A_{i} x(k)+B_{i} u(k)+F_{i} f(k)\right) \\
y(k)=C x(k)
\end{array}\right.
$$

where $x(k) \in \mathbb{R}^{n}$ is the descriptor state vector, $u(k) \in \mathbb{R}^{p}$ is the input vector, $y(k) \in \mathbb{R}^{m}$ is the output vector and $f(k) \in \mathbb{R}^{q}$ represents the fault vector. $E \in \mathbb{R}^{n \times n}$ is a constant matrix which may be rank deficient, i.e. $\operatorname{rank}(E)=r \leq n . A_{i} \in \mathbb{R}^{n \times n}, B_{i} \in \mathbb{R}^{n \times p}, F_{i} \in \mathbb{R}^{n \times q}$ and $C \in \mathbb{R}^{m \times m}$ are known constant matrices, and it is assumed that $\operatorname{rank}(C)=m, \operatorname{rank}\left(F_{i}\right)=q, q \leq m$. In system (1), $\theta(k) \in \mathbb{R}^{l}$ is a bounded time-varying parameter vector which is assumed to be measured online and not affected by fault, which is a general assumption in the LPV framework [9, 11]. System (1) refers to a polytopic LPV descritptor system which is a particular class of LPV systems and $\rho_{i}(\theta(k)), i=1,2, \ldots, h$ are the weighting functions. It is assumed that the weighting functions 
$\rho_{i}(\theta(k)), i=1,2, \ldots, h$ vary within the following convex set $\Omega$ for all $k$ :

$$
\Omega=\left\{\rho(\theta(k)) \in \mathbb{R}^{h}, \rho(\theta(k))=\left[\rho_{1}(\theta(k)), \ldots, \rho_{h}(\theta(k))\right]^{T}, \rho_{i}(\theta(k)) \geq 0, \forall i, \sum_{i=1}^{h} \rho_{i}(\theta(k))=1\right\}
$$

Herein, $h$ is the total number of weighting functions. It is noted that $h$ is determined in the modeling procedure of LPV system (1). For instance, if LPV system (1) is obtained by interpolation in a given polytope, then $h$ equals to the number of extreme values of this polytope (see [19] for more details about constructing a polytopic LPV system). If (1) is modeled by other methodologies, $h$ depends on specific modeling technique.

In order to achieve fault estimation, the following assumptions are considered in this paper.

\section{Assumption 1}

Matrices $E$ and $C$ satisfy the following rank condition

$$
\operatorname{rank}\left[\begin{array}{c}
E \\
C
\end{array}\right]=n
$$

\section{Assumption 2}

The fault $f(k)$ in (1) is piecewise constant or slow varying, i.e. it is assumed that

$$
f(k+1)=f(k)
$$

\section{Remark 1}

It should be noted that Assumption 2 is just a theoretical condition for the convenience of design that is often used in the literature, see e.g. [20, 23]. Moreover, this assumption will be relaxed in Section 4.

By defining the following augmented state vector

$$
\bar{x}(k)=\left[\begin{array}{l}
x(k) \\
f(k)
\end{array}\right]
$$

an augmented system is constructed as follows

$$
\left\{\begin{array}{l}
\bar{E} \bar{x}(k+1)=\sum_{i=1}^{h} \rho_{i}(\theta(k))\left(\bar{A}_{i} \bar{x}(k)+\bar{B}_{i} u(k)\right) \\
y(k)=\bar{C} \bar{x}(k)
\end{array}\right.
$$


where

$$
\bar{E}=\left[\begin{array}{cc}
E & 0 \\
0 & I_{q}
\end{array}\right], \bar{A}_{i}=\left[\begin{array}{cc}
A_{i} & F_{i} \\
0 & I_{q}
\end{array}\right], \bar{B}_{i}=\left[\begin{array}{c}
B_{i} \\
0
\end{array}\right], \bar{C}=\left[\begin{array}{ll}
C & 0
\end{array}\right]
$$

Following the definition of $\bar{x}(k)$ in (5), the state vector $x(k)$ and fault $f(k)$ in the system (1) can be estimated simultaneously if the estimation of augmented state $\bar{x}(k)$ is obtained. Therefore, the fault estimation problem for system (1) is converted as an observer design for the augmented system (6).

\section{ACTUATOR FAULT ESTIMATION OBSERVER DESIGN}

For the augmented system (6), the following observer is constructed

$$
\left\{\begin{array}{l}
\xi(k+1)=\sum_{i=1}^{h} \rho_{i}(\theta(k))\left(T \bar{A}_{i} \hat{\bar{x}}(k)+T \bar{B}_{i} u(k)+L_{i}(y(k)-\bar{C} \hat{\bar{x}}(k))\right) \\
\hat{\bar{x}}(k)=\xi(k)+N y(k)
\end{array}\right.
$$

where $\xi(k) \in \mathbb{R}^{(n+q)}$ is an intermediate state vector, $\hat{\bar{x}}(k) \in \mathbb{R}^{(n+q)}$ is the estimate of the augmented state $\bar{x}(k) . T \in \mathbb{R}^{(n+q) \times n}, N \in \mathbb{R}^{(n+q) \times m}$ and $L_{i} \in \mathbb{R}^{(n+q) \times m}, i=1,2, \ldots, h$ are matrices to be synthesized.

The following Lemma will be used in the sequel.

Lemma 1

Given matrices $\mathscr{X} \in \mathbb{R}^{a \times b}, \mathscr{B} \in \mathbb{R}^{b \times c}$, and $\mathscr{Y} \in \mathbb{R}^{a \times c}$. If $\operatorname{rank}(\mathscr{B})=c$, then the general solution of the following equation

$$
\mathscr{X} \mathscr{B}=\mathscr{Y}
$$

is given by

$$
\mathscr{X}=\mathscr{Y} \mathscr{B}^{\dagger}+\mathscr{S}\left(I_{b}-\mathscr{B} \mathscr{B}^{\dagger}\right)
$$

where $\mathscr{S} \in \mathbb{R}^{a \times b}$ is an arbitrary matrix. Herein and throughout this paper, the symbol ${ }^{\dagger}$ denotes the pseudo-inverse of a matrix.

Proof

Lemma 1 is a straightforward result of the Theorem of Penrose ([24]). 
Now, the following Theorem is proposed to design the observer (8).

\section{Theorem 1}

The dynamic system (8) is an observer for the augmented system (6) if there exist a symmetric positive definite matrix $P \in \mathbb{R}^{(n+q) \times(n+q)}$, a matrix $Y \in \mathbb{R}^{(n+q) \times(n+q+m)}$ and matrices $W_{i} \in$ $\mathbb{R}^{(n+q) \times m}, i=1,2, \ldots, h$ such that the following LMIs hold

$$
\left[\begin{array}{cc}
-P & \mathscr{A}_{1 i}^{T} P+\mathscr{A}_{2 i}^{T} Y^{T}-\bar{C}^{T} W_{i}^{T} \\
* & -P
\end{array}\right]<0, i=1,2, \ldots, h
$$

Herein and throughout this paper, $*$ is used to represent the elements induced by symmetry. In (11), matrices $\mathscr{A}_{1 i}$ and $\mathscr{A}_{2 i}$ are

$$
\mathscr{A}_{1 i}=\Psi^{\dagger} \alpha_{1} \bar{A}_{i}, \mathscr{A}_{2 i}=\left(I_{n+q+m}-\Psi \Psi^{\dagger}\right) \alpha_{1} \bar{A}_{i}
$$

where $\Psi \in \mathbb{R}^{(n+q+m) \times(n+q)}$ is given by

$$
\Psi=\left[\begin{array}{c}
\bar{E} \\
\bar{C}
\end{array}\right]
$$

Moreover, if the LMIs in (11) are solved, the matrices $T, N$ and $L_{i}, i=1,2, \ldots, h$ in observer (8) are determined by

$$
\begin{gathered}
T=\Psi^{\dagger} \alpha_{1}+P^{-1} Y\left(I_{n+q+m}-\Psi \Psi^{\dagger}\right) \alpha_{1} \\
N=\Psi^{\dagger} \alpha_{2}+P^{-1} Y\left(I_{n+q+m}-\Psi \Psi^{\dagger}\right) \alpha_{2} \\
L_{i}=P^{-1} W_{i}, i=1,2, \ldots, h
\end{gathered}
$$

where $\alpha_{1} \in \mathbb{R}^{(n+q+m) \times(n+q)}, \alpha_{2} \in \mathbb{R}^{(n+q+m) \times m}$ are given by

$$
\alpha_{1}=\left[\begin{array}{c}
I_{n+q} \\
0
\end{array}\right], \alpha_{2}=\left[\begin{array}{c}
0 \\
I_{m}
\end{array}\right]
$$

Proof

Under the Assumption 1, it is easy to derived that

$$
\operatorname{rank}\left[\begin{array}{c}
\bar{E} \\
\bar{C}
\end{array}\right]=\operatorname{rank}\left[\begin{array}{cc}
E & 0 \\
0 & I_{q} \\
C & 0
\end{array}\right]=n+q
$$


Then, according to Lemma 1, there exist matrices $T$ and $N$ such that the following equation

$$
T \bar{E}+N \bar{C}=I_{n+q}
$$

holds.

Equation (19) can be written as

$$
\left[\begin{array}{ll}
T & N
\end{array}\right]\left[\begin{array}{l}
\bar{E} \\
\bar{C}
\end{array}\right]=I_{n+q}
$$

By using Lemma 1 to (20), the general solution for $\left[\begin{array}{ll}T & N\end{array}\right]$ is given by

$$
\left[\begin{array}{ll}
T & N
\end{array}\right]=\left[\begin{array}{l}
\bar{E} \\
\bar{C}
\end{array}\right]^{\dagger}+S\left(I_{n+q+m}-\left[\begin{array}{l}
\bar{E} \\
\bar{C}
\end{array}\right]\left[\begin{array}{l}
\bar{E} \\
\bar{C}
\end{array}\right]^{\dagger}\right)
$$

where $S \in \mathbb{R}^{(n+q) \times(n+q+m)}$ is an arbitrary matrix.

Following (21), equation (19) holds if the matrices $T$ and $N$ are respectively determined by

$$
T=\Psi^{\dagger} \alpha_{1}+S\left(I_{n+q+m}-\Psi \Psi^{\dagger}\right) \alpha_{1}
$$

and

$$
N=\Psi^{\dagger} \alpha_{2}+S\left(I_{n+q+m}-\Psi \Psi^{\dagger}\right) \alpha_{2}
$$

where $\Psi, \alpha_{1}$ and $\alpha_{2}$ are given by (13) and (17).

Now, define the augmented state estimation error as

$$
e(k)=\bar{x}(k)-\hat{\bar{x}}(k)
$$

Using equation (19), the state estimation error is written as

$$
\begin{aligned}
e(k) & =\bar{x}(k)-\xi(k)-N y(k) \\
& =\left(I_{n+q}-N \bar{C}\right) \bar{x}(k)-\xi(k) \\
& =T \bar{E} \bar{x}(k)-\xi(k)
\end{aligned}
$$

Taking the following Lyapunov function

$$
V(k)=e^{T}(k) P e(k), P>0
$$


Then, the time difference of $V(k)$ is

$$
\begin{aligned}
\Delta V(k)= & V(k+1)-V(k) \\
= & e^{T}(k+1) P e(k+1)-e^{T}(k) P e(k) \\
= & e^{T}(k)\left\{\sum_{i=1}^{h} \rho_{i}(\theta(k))\left(T \bar{A}_{i}-L_{i} \bar{C}\right)\right\}^{T} P\left\{\sum_{i=1}^{h} \rho_{i}(\theta(k))\left(T \bar{A}_{i}-L_{i} \bar{C}\right)\right\} e(k) \\
& -e^{T}(k) P e(k)
\end{aligned}
$$

From (27), it can be seen that $\Delta V(k)<0$ holds if

$$
\left\{\sum_{i=1}^{h} \rho_{i}(\theta(k))\left(T \bar{A}_{i}-L_{i} \bar{C}\right)\right\}^{T} P\left\{\sum_{i=1}^{h} \rho_{i}(\theta(k))\left(T \bar{A}_{i}-L_{i} \bar{C}\right)\right\}-P<0
$$

By Schur complement Lemma [25], inequality (28) is equivalent to

$$
\left[\begin{array}{cc}
-P & \sum_{i=1}^{h} \rho_{i}(\theta(k))\left(T \bar{A}_{i}-L_{i} \bar{C}\right)^{T} P \\
* & -P
\end{array}\right]<0
$$

Using the property of $\rho_{i}(\theta(k))$ in (2), it can be obtained that the inequality (29) holds if

$$
\left[\begin{array}{cc}
-P & \left(T \bar{A}_{i}-L_{i} \bar{C}\right)^{T} P \\
* & -P
\end{array}\right]<0, i=1,2 \ldots, h
$$

is fulfilled.

Substituting (22) into (30) and considering the definitions of $\mathscr{A}_{1 i}$ and $\mathscr{A}_{2 i}$ in (12), the inequalities in (30) become

$$
\left[\begin{array}{cc}
-P & \left(\mathscr{A}_{1 i}+S \mathscr{A}_{2 i}-L_{i} \bar{C}\right)^{T} P \\
* & -P
\end{array}\right]<0, i=1,2, \ldots, h
$$

By letting $Y=P S$ and $W_{i}=P L_{i}$, the equalities in (31) become the LMIs in (11). Moreover, if the LMIs in (11) are solved, the matrices $L_{i}, \mathrm{i}=1,2, \ldots, \mathrm{h}$ can be determined by $L_{i}=P^{-1} W_{i}$ and the matrix $S$ is obtained by $S=P^{-1} Y$. Then, the matrices $T$ and $N$ can be determined by (14) and (15), respectively.

\section{Remark 2}

Once the augmented state estimation $\hat{\bar{x}}(k)$ is obtained, the fault estimation $\hat{f}(k)$ can be calculated by

$$
\hat{f}(k)=C_{f} \hat{\bar{x}}(k)
$$


where $C_{f} \in \mathbb{R}^{q \times(n+q)}$ is given by

$$
C_{f}=\left[\begin{array}{ll}
0 & I_{q}
\end{array}\right]
$$

\section{ROBUST FAULT ESTIMATION OBSERVER DESIGN}

In Section 3, a fault estimation observer design method is proposed. However, the method proposed in Section 3 assumes the fault to be constant, which is a restrictive assumption in some practical situations. Moreover, model uncertainty and measurement noise are not considered in Section 2. To overcome these problems, this section proposes a robust fault estimation observer design method to relax Assumption 2 and attenuate the effect of disturbance and noise.

Consider the following polytopic LPV descriptor system

$$
\left\{\begin{array}{l}
E x(k+1)=\sum_{i=1}^{h} \rho_{i}(\theta(k))\left(A_{i} x(k)+B_{i} u(k)+F_{i} f(k)+D_{i} w(k)\right) \\
y(k)=C x(k)+v(k)
\end{array}\right.
$$

where $w(k) \in \mathbb{R}^{d}$ represents the unknown disturbance, $D_{i} \in \mathbb{R}^{n \times d}$ is the distribution matrix, and $v(k) \in \mathbb{R}^{m}$ is the measurement noise. Other symbols in (34) are the same as defined before.

For the descriptor system (34), an augmented system is constructed as follows

$$
\left\{\begin{array}{l}
\bar{E} \bar{x}(k+1)=\sum_{i=1}^{h} \rho_{i}(\theta(k))\left(\bar{A}_{i} \bar{x}(k)+\bar{B}_{i} u(k)+\bar{D}_{i} w(k)+\bar{G}_{i} \Delta f(k)\right) \\
y(k)=\bar{C} \bar{x}(k)+v(k)
\end{array}\right.
$$

where

$$
\begin{gathered}
\bar{x}(k)=\left[\begin{array}{c}
x(k) \\
f(k)
\end{array}\right], \Delta f(k)=f(k+1)-f(k) \\
\bar{E}=\left[\begin{array}{cc}
E & 0 \\
0 & I_{q}
\end{array}\right], \bar{A}_{i}=\left[\begin{array}{cc}
A_{i} & F_{i} \\
0 & I_{q}
\end{array}\right], \bar{B}_{i}=\left[\begin{array}{c}
B_{i} \\
0
\end{array}\right], \bar{D}_{i}=\left[\begin{array}{c}
D_{i} \\
0
\end{array}\right], \bar{G}_{i}=\left[\begin{array}{l}
0 \\
I_{q}
\end{array}\right], \bar{C}=\left[\begin{array}{ll}
C & 0
\end{array}\right]
\end{gathered}
$$

Then, the following Theorem is proposed to design a robust observer in the form of (8) for the augmented system (35).

\section{Theorem 2}

For the augmented system (35) and given scalars $\gamma_{d}>0, \gamma_{f}>0 \gamma_{L}>0 \gamma_{N}>0$, if there exist a 
symmetric positive definite matrix $P \in \mathbb{R}^{(n+q) \times(n+q)}$, a matrix $Y \in \mathbb{R}^{(n+q) \times(n+q+m)}$ and matrices $W_{i} \in \mathbb{R}^{(n+q) \times m}, i=1,2, \ldots, h$ such that the following LMIs hold $\left[\begin{array}{cccccc}-P+C_{f}^{T} C_{f} & 0 & 0 & 0 & 0 & \mathscr{A}_{1 i}^{T} P+\mathscr{A}_{2 i}^{T} Y^{T}-\bar{C}^{T} W_{i}^{T} \\ * & -\gamma_{d}^{2} I_{d} & 0 & 0 & 0 & \mathscr{D}_{1 i}^{T} P+\mathscr{D}_{2 i}^{T} Y^{T} \\ * & * & -\gamma_{f}^{2} I_{q} & 0 & 0 & \mathscr{G}_{1 i}^{T} P+\mathscr{G}_{2 i}^{T} Y^{T} \\ * & * & * & -\gamma_{L}^{2} I_{m} & 0 & -W_{i}^{T} \\ * & * & * & * & -\gamma_{N}^{2} I_{m} & -\mathscr{N}_{1}^{T} P-\mathscr{N}_{2}^{T} Y^{T} \\ * & * & * & * & * & -P\end{array}\right]<0, i=1,2, \ldots, h$

where $\mathscr{A}_{1 i}$ and $\mathscr{A}_{2 i}$ are given in (12) and

$$
\begin{gathered}
\mathscr{D}_{1 i}=\Psi^{\dagger} \alpha_{1} \bar{D}_{i}, \mathscr{D}_{2 i}=\left(I_{n+q+m}-\Psi \Psi^{\dagger}\right) \alpha_{1} \bar{D}_{i} \\
\mathscr{G}_{1 i}=\Psi^{\dagger} \alpha_{1} \bar{G}_{i}, \mathscr{G}_{2 i}=\left(I_{n+q+m}-\Psi \Psi^{\dagger}\right) \alpha_{1} \bar{G}_{i} \\
\mathscr{N}_{1}=\Psi^{\dagger} \alpha_{2}, \mathscr{N}_{2}=\left(I_{n+q+m}-\Psi \Psi^{\dagger}\right) \alpha_{2}
\end{gathered}
$$

Then the fault estimation error $e_{f}(k)=f(k)-\hat{f}(k)$ is robust against the disturbance, fault variation, and measurement noise, i.e.

$$
\left\|e_{f}\right\|_{2} \leq \sqrt{\gamma_{d}^{2}\|w\|_{2}^{2}+\gamma_{f}^{2}\|\Delta f\|_{2}^{2}+\left(\gamma_{L}^{2}+\gamma_{N}^{2}\right)\|v\|_{2}^{2}+V(0)}
$$

where $\|\cdot\|_{2}$ represents the $\mathscr{L}_{2}$ norm, and $V(0)$ is a quadratic function of $e(0)$ which will be given later. Moreover, if the LMIs in (38) are solved, the matrices $T, N$ and $L_{i}, i=1,2, \ldots, h$ are determined by

$$
\begin{gathered}
T=\Psi^{\dagger} \alpha_{1}+P^{-1} Y\left(I_{n+q+m}-\Psi \Psi^{\dagger}\right) \alpha_{1} \\
N=\Psi^{\dagger} \alpha_{2}+P^{-1} Y\left(I_{n+q+m}-\Psi \Psi^{\dagger}\right) \alpha_{2} \\
L_{i}=P^{-1} W_{i}, i=1,2, \ldots, h
\end{gathered}
$$

where $\Psi, \alpha_{1}$ and $\alpha_{2}$ are given in (13) and (17). 
Proof

If the matrices $T$ and $N$ are respectively determined by

$$
T=\Psi^{\dagger} \alpha_{1}+S\left(I_{n+q+m}-\Psi \Psi^{\dagger}\right) \alpha_{1}
$$

and

$$
N=\Psi^{\dagger} \alpha_{2}+S\left(I_{n+q+m}-\Psi \Psi^{\dagger}\right) \alpha_{2}
$$

then the following equation holds

$$
T \bar{E}+N \bar{C}=I_{n+q}
$$

Following (48) and using (25), (35) and (8), it is easy to derive the following error dynamics

$$
\begin{aligned}
e(k+1)= & T \bar{E} \bar{x}(k+1)-\xi(k+1)-N v(k+1) \\
& -N v(k+1) \\
= & \sum_{i=1}^{h} \rho_{i}(\theta(k))\left(\left(T \bar{A}_{i}-L_{i} \bar{C}\right) e(k)+T \bar{D}_{i} w(k)+T \bar{G}_{i} \Delta f(k)-L_{i} v(k)\right. \\
& -N v(k+1))
\end{aligned}
$$

Take the following Lyapunov function

$$
V(k)=e^{T}(k) P e(k), P>0
$$

and define the following criterion function

$$
\begin{aligned}
J= & \sum_{k=0}^{\infty}\left(\Delta V(k)+e_{f}^{T}(k) e_{f}(k)-\gamma_{d}^{2} w^{T}(k) w(k)-\gamma_{f}^{2}(\Delta f(k))^{T} \Delta f(k)\right. \\
& \left.-\gamma_{L}^{2} v^{T}(k) v(k)-\gamma_{N}^{2} v^{T}(k+1) v(k+1)\right)
\end{aligned}
$$

By using the fact that $\Delta V(k)=V(k+1)-V(k)$, we obtain

$$
\begin{aligned}
J= & V(\infty)-V(0)+\sum_{k=0}^{\infty}\left(e_{f}^{T}(k) e_{f}(k)-\gamma^{2} w^{T}(k) w(k)-\gamma_{f}^{2}(\Delta f(k))^{T} \Delta f(k)\right. \\
& \left.-\gamma_{L}^{2} v^{T}(k) v(k)-\gamma_{N}^{2} v^{T}(k+1) v(k+1)\right)
\end{aligned}
$$

Since $V(\infty) \geq 0$, the inequality $J<0$ implies

$$
\left\|e_{f}\right\|_{2}^{2}-\gamma_{d}^{2}\|w\|_{2}^{2}-\gamma_{f}^{2}\|\Delta f\|_{2}^{2}-\gamma_{L}^{2}\|v\|_{2}^{2}-\gamma_{N}^{2}\|v\|_{2}^{2}-V(0)<0
$$

i.e., the criteria (42) is satisfied. 
On the other hand, it is clear that $J<0$ holds if

$$
\begin{gathered}
\Delta V(k)+e_{f}^{T}(k) e_{f}(k)-\gamma_{d}^{2} w^{T}(k) w(k)-\gamma_{f}^{2}(\Delta f(k))^{T} \Delta f(k) \\
-\gamma_{L}^{2} v^{T}(k) v(k)-\gamma_{N}^{2} v^{T}(k+1) v(k+1)<0
\end{gathered}
$$

is fulfilled for all $k$. Therefore, the criteria (42) is satisfied if (54) holds.

Using (49) yields

$$
\begin{aligned}
\Delta & V(k)+e_{f}^{T}(k) e_{f}(k)-\gamma_{d}^{2} w^{T}(k) w(k)-\gamma_{f}^{2}(\Delta f(k))^{T} \Delta f(k)-\gamma_{L}^{2} v^{T}(k) v(k)-\gamma_{N}^{2} v^{T}(k+1) v(k+1) \\
= & \left\{\sum_{i=1}^{h} \rho_{i}(\theta(k))\left(\left(T \bar{A}_{i}-L_{i} \bar{C}\right) e(k)+T \bar{D}_{i} w(k)+T \bar{G}_{i} \Delta f(k)-L_{i} v(k)-N v(k+1)\right)\right\}^{T} P \\
& \times\left\{\sum_{i=1}^{h} \rho_{i}(\theta(k))\left(\left(T \bar{A}_{i}-L_{i} \bar{C}\right) e(k)+T \bar{D}_{i} w(k)+T \bar{G}_{i} \Delta f(k)-L_{i} v(k)-N v(k+1)\right)\right\} \\
& -e^{T}(k) P e(k)+e_{f}^{T}(k) e_{f}(k)-\gamma_{d}^{2} w^{T}(k) w(k)-\gamma_{f}^{2}(\Delta f(k))^{T} \Delta f(k)-\gamma_{L}^{2} v^{T}(k) v(k) \\
& -\gamma_{N}^{2} v^{T}(k+1) v(k+1) \\
\triangleq & {\left[\begin{array}{c}
e(k) \\
w(k) \\
\Delta f(k) \\
v(k) \\
v(k+1)
\end{array}\right]\left[\begin{array}{ccccc}
\Omega_{11} & \Omega_{12} & \Omega_{13} & \Omega_{14} & \Omega_{15} \\
* & \Omega_{22} & \Omega_{23} & \Omega_{24} & \Omega_{25} \\
* & * & \Omega_{33} & \Omega_{34} & \Omega_{35} \\
* & * & * & \Omega_{44} & \Omega_{45} \\
* & * & * & * & \Omega_{55}
\end{array}\right]\left[\begin{array}{c}
e(k) \\
w(k) \\
\Delta f(k) \\
v(k) \\
v(k+1)
\end{array}\right] }
\end{aligned}
$$

where

$$
\begin{gathered}
\Omega_{11}=\left\{\sum_{i=1}^{h} \rho_{i}(\theta(k))\left(T \bar{A}_{i}-L_{i} \bar{C}\right)\right\}^{T} P\left\{\sum_{i=1}^{h} \rho_{i}(\theta(k))\left(T \bar{A}_{i}-L_{i} \bar{C}\right)\right\}-P+C_{f}^{T} C_{f} \\
\Omega_{12}=\left\{\sum_{i=1}^{h} \rho_{i}(\theta(k))\left(T \bar{A}_{i}-L_{i} \bar{C}\right)\right\}^{T} P\left\{\sum_{i=1}^{h} \rho_{i}(\theta(k))\left(T \bar{D}_{i}\right)\right\} \\
\Omega_{13}=\left\{\sum_{i=1}^{h} \rho_{i}(\theta(k))\left(T \bar{A}_{i}-L_{i} \bar{C}\right)\right\}^{T} P\left\{\sum_{i=1}^{h} \rho_{i}(\theta(k))\left(T \bar{G}_{i}\right)\right\} \\
\Omega_{14}=\left\{\sum_{i=1}^{h} \rho_{i}(\theta(k))\left(T \bar{A}_{i}-L_{i} \bar{C}\right)\right\}^{T} P\left\{-\sum_{i=1}^{h} \rho_{i}(\theta(k)) L_{i}\right\} \\
\Omega_{15}=\left\{\sum_{i=1}^{h} \rho_{i}(\theta(k))\left(T \bar{A}_{i}-L_{i} \bar{C}\right)\right\}^{T} P\left\{-\sum_{i=1}^{h} \rho_{i}(\theta(k)) N\right\} \\
\Omega_{22}=\left\{\sum_{i=1}^{h} \rho_{i}(\theta(k))\left(T \bar{D}_{i}\right)\right\}^{T} P\left\{\sum_{i=1}^{h} \rho_{i}(\theta(k))\left(T \bar{D}_{i}\right)\right\}-\gamma_{d}^{2} I_{d}
\end{gathered}
$$




$$
\begin{gathered}
\Omega_{23}=\left\{\sum_{i=1}^{h} \rho_{i}(\theta(k))\left(T \bar{D}_{i}\right)\right\}^{T} P\left\{\sum_{i=1}^{h} \rho_{i}(\theta(k))\left(T \bar{G}_{i}\right)\right\} \\
\Omega_{24}=\left\{\sum_{i=1}^{h} \rho_{i}(\theta(k))\left(T \bar{D}_{i}\right)\right\}^{T} P\left\{-\sum_{i=1}^{h} \rho_{i}(\theta(k)) L_{i}\right\} \\
\Omega_{25}=\left\{\sum_{i=1}^{h} \rho_{i}(\theta(k))\left(T \bar{D}_{i}\right)\right\}^{T} P\left\{-\sum_{i=1}^{h} \rho_{i}(\theta(k)) N\right\} \\
\Omega_{33}=\left\{\sum_{i=1}^{h} \rho_{i}(\theta(k))\left(T \bar{G}_{i}\right)\right\}^{T} P\left\{\sum_{i=1}^{h} \rho_{i}(\theta(k))\left(T \bar{G}_{i}\right)\right\}-\gamma_{f}^{2} I_{q} \\
\Omega_{34}=\left\{\sum_{i=1}^{h} \rho_{i}(\theta(k))\left(T \bar{G}_{i}\right)\right\}^{T} P\left\{-\sum_{i=1}^{h} \rho_{i}(\theta(k)) L_{i}\right\} \\
\Omega_{44}=\left\{\sum_{i=1}^{h} \rho_{i}(\theta(k)) L_{i}\right\}^{T} P\left\{\sum_{i=1}^{h} \rho_{i}(\theta(k)) L_{i}\right\}-\gamma_{L}^{2} I_{m} \\
\left.\Omega_{i=1}^{h}-\rho_{i}(\theta(k)) L_{i}\right\}^{T} P\left\{-\sum_{i=1}^{h} \rho_{i}(\theta(k)) N\right\} \\
\left.\sum_{i=1}^{h} \rho_{i}(\theta(k))\left(T \bar{G}_{i}\right)\right\}^{T} P\left\{-\sum_{i=1}^{h} \rho_{i}(\theta(k)) N\right\}
\end{gathered}
$$

Herein, the equation $e_{f}(k)=C_{f} e(k)$ is used.

From (55), it can be seen that (54) holds if

$$
\left[\begin{array}{ccccc}
\Omega_{11} & \Omega_{12} & \Omega_{13} & \Omega_{14} & \Omega_{15} \\
* & \Omega_{22} & \Omega_{23} & \Omega_{24} & \Omega_{25} \\
* & * & \Omega_{33} & \Omega_{34} & \Omega_{35} \\
* & * & * & \Omega_{44} & \Omega_{45} \\
* & * & * & * & \Omega_{55}
\end{array}\right]<0
$$


By using Schur complement Lemma [25], the inequality (71) is equivalent to

$$
\left[\begin{array}{cccccc}
-P+C_{f}^{T} C_{f} & 0 & 0 & 0 & 0 & \sum_{i=1}^{h} \rho_{i}(\theta(k))\left(T \bar{A}_{i}-L_{i} \bar{C}\right)^{T} P \\
* & -\gamma_{d}^{2} I_{d} & 0 & 0 & 0 & \sum_{i=1}^{h} \rho_{i}(\theta(k))\left(T \bar{D}_{i}\right)^{T} P \\
* & * & -\gamma_{f}^{2} I_{q} & 0 & 0 & \sum_{i=1}^{h} \rho_{i}(\theta(k))\left(T \bar{G}_{i}\right)^{T} P \\
* & * & * & -\gamma_{L}^{2} I_{q} & 0 & -\sum_{i=1}^{h} \rho_{i}(\theta(k)) L_{i}^{T} P \\
* & * & * & * & -\gamma_{N}^{2} I_{q} & -\sum_{i=1}^{h} \rho_{i}(\theta(k)) N^{T} P \\
* & * & * & * & * & -P
\end{array}\right]<0
$$

Using the property of $\rho_{i}(\theta(k))$ in (2), the inequality (72) holds if

$$
\left[\begin{array}{cccccc}
-P+C_{f}^{T} C_{f} & 0 & 0 & 0 & 0 & \left(T \bar{A}_{i}-L_{i} \bar{C}\right)^{T} P \\
* & -\gamma_{d}^{2} I_{d} & 0 & 0 & 0 & \left(T \bar{D}_{i}\right)^{T} P \\
* & * & -\gamma_{f}^{2} I_{q} & 0 & 0 & \left(T \bar{G}_{i}\right)^{T} P \\
* & * & * & -\gamma_{L}^{2} I_{m} & 0 & -L_{i}^{T} P \\
* & * & * & * & -\gamma_{N}^{2} I_{m} & -N^{T} P \\
* & * & * & * & * & -P
\end{array}\right]<0, i=1,2, \ldots, h
$$

Substituting (46) and (47) into (73) and using the definitions of $\mathscr{A}_{1 i}, \mathscr{A}_{2 i}, \mathscr{D}_{1 i}, \mathscr{D}_{2 i}, \mathscr{G}_{1 i}, \mathscr{G}_{2 i}, \mathscr{N}_{1}$, $\mathscr{N}_{2}$ in (12) and (39)-(41), we obtain

$$
\left[\begin{array}{cccccc}
-P+C_{f}^{T} C_{f} & 0 & 0 & 0 & 0 & \mathscr{A}_{1 i}^{T} P+\mathscr{A}_{2 i}^{T} S^{T} P-\bar{C}^{T} L_{i}^{T} P \\
* & -\gamma_{d}^{2} I_{d} & 0 & 0 & 0 & \mathscr{D}_{1 i}^{T} P+\mathscr{D}_{2 i}^{T} S^{T} P \\
* & * & -\gamma_{f}^{2} I_{q} & 0 & 0 & \mathscr{G}_{1 i}^{T} P+\mathscr{G}_{2 i}^{T} S^{T} P \\
* & * & * & -\gamma_{L}^{2} I_{m} & 0 & -L_{i}^{T} P \\
* & * & * & * & -\gamma_{N}^{2} I_{m} & -\mathscr{N}_{1}^{T} P+\mathscr{N}_{2}^{T} S^{T} P \\
* & * & * & * & * & -P \\
i=1,2, \ldots, h & & & &
\end{array}\right]<0,
$$

By letting $Y=P S$ and $W_{i}=P L_{i}$, the inequalities in (74) become the LMIs in (38). 


\section{Remark 3}

Reference [26] has proposed an LMI-based method to design unknown input observer (UIO) for Takagi-Sugeno descriptor systems. In [26], the parameter matrices of the UIO can also be determined by solving LMIs. However, it should be pointed out that this paper is different from [26] in the following aspects. In [26], an unknown input observer design method is proposed and applied to fault detection and isolation. In this paper, an augmented observer is designed and used for fault estimation. The proposed method is able not only to detect the occurrence of fault, but also to estimate the fault magnitude. Moreover, this paper also deals with the effect of the measurement noise, which is not considered in [26].

\section{Remark 4}

In dealing with time-varying faults, there may be a time-delay between the fault estimation and the actual fault. This phenomenon results from the influence of fault variation. Theoretically, the attenuation level $\gamma_{f}$ can be minimized so that the fault estimation is insenstive to the fault variation. However, the cost is that the fault estimation becomes less robust to disturbance and measurement noise. Therefore, the choice of $\gamma_{d}, \gamma_{f}, \gamma_{L}$ and $\gamma_{N}$ is a trade-off.

\section{SIMULATIONS}

In this section, a truck-trailer model [27] is used to show the effectiveness of the proposed method.

From [27], the dynamic equation of a truck-trailer model is given by

$$
\left\{\begin{array}{l}
x_{1}(k+1)=x_{1}(k)+\frac{\theta(k) t}{l} \tan (u(k))-\frac{\theta(k) t}{L} \sin \left(x_{1}(k)\right) \\
x_{2}(k+1)=x_{2}(k)+\frac{\theta(k) t}{L} \sin \left(x_{1}(k)\right) \\
x_{3}(k+1)=x_{3}(k)+\theta(k) t \cos \left(x_{1}(k)\right)\left(\sin \frac{x_{2}(k+1)+x_{2}(k)}{2}\right)
\end{array}\right.
$$

where $x_{1}(k)$ is the angle difference between truck and trailer, $x_{2}(k)$ is the angle of trailer, $x_{3}(k)$ is the vertical position of rear end of trailer and $u(k)$ is the steering angle. $l$ is the length of truck, $L$ is the length of trailer, $t$ is sampling time and $\theta(k)$ is the speed of backing up. In this paper, $l=2.8 \mathrm{~m}$, $L=5.5 \mathrm{~m}, t=2 \mathrm{~s}$. It is assumed that the speed $v$ varies according to $\theta(k) \in[-1.2,-0.6]$. 
Similar to [27], it is assumed that $x_{1}(k)$ is small. Then, the truck-trailer model (75) can be simplified as

$$
\left\{\begin{array}{l}
x_{1}(k+1)=\left(1-\frac{\theta(k) t}{L}\right) x_{1}(k)+\frac{\theta(k) t}{l} \tan (u(k)) \\
x_{2}(k+1)=x_{2}(k)+\frac{\theta(k) t}{L} x_{1}(k) \\
x_{3}(k+1)=x_{3}(k)+\theta(k) t \cdot \sin \left(\frac{x_{2}(k+1)+x_{2}(k)}{2}\right)
\end{array}\right.
$$

In order to obtain an LPV descriptor representation, we introduce the following variables

$$
x_{4}(k)=\frac{\theta(k) t}{2} x_{2}(k), \tilde{u}(k)=\tan (u(k))
$$

Then, the simplified track-trailer model (76) can be written as follows

$$
\left\{\begin{array}{l}
E x(k+1)=A(\theta(k))+B(\theta(k)) \tilde{u}(k)+D(\theta(k)) w(k) \\
y(k)=C x(k)
\end{array}\right.
$$

where

$$
\begin{gathered}
E=\left[\begin{array}{llll}
1 & 0 & 0 & 0 \\
0 & 1 & 0 & 0 \\
0 & 0 & 1 & -1 \\
0 & 0 & 0 & 0
\end{array}\right], A(\theta(k))=\left[\begin{array}{cccc}
1-\frac{\theta(k) t}{L} & 0 & 0 & 0 \\
\frac{\theta(k) t}{L} & 1 & 0 & 0 \\
0 & 0 & 1 & 1 \\
0 & -\frac{\theta(k) t}{2} & 0 & 1
\end{array}\right], B(\theta(k))=\left[\begin{array}{c}
\frac{\theta(k) t}{l} \\
0 \\
0 \\
0
\end{array}\right] \\
D(\theta(k))=\left[\begin{array}{c}
0 \\
0 \\
\theta(k) t \\
0
\end{array}\right], C=\left[\begin{array}{llll}
0 & 1 & 0 & 0 \\
0 & 0 & 1 & 0 \\
0 & 0 & 0 & 1
\end{array}\right], w(k)=\sin \left(\frac{x_{2}(k+1)+x_{2}(k)}{2}\right)-\frac{x_{2}(k+1)+x_{2}(k)}{2}
\end{gathered}
$$

Then, the LPV descriptor system (77) is approximated by the following polytopic representation

$$
\left\{\begin{array}{l}
E x(k+1)=\sum_{i=1}^{2} \rho_{i}(\theta(k))\left(A_{i} x(k)+B_{i} \tilde{u}(k)+F_{i} f(k)+D_{i} w(k)\right) \\
y(k)=C x(k)
\end{array}\right.
$$


The obtained system evolves in a polytopic of two vertices correspending to the extreme values of $v$. The matrices in system (78) are given as

$$
\begin{gathered}
E=\left[\begin{array}{llll}
1 & 0 & 0 & 0 \\
0 & 1 & 0 & 0 \\
0 & 0 & 1 & -1 \\
0 & 0 & 0 & 0
\end{array}\right], A_{1}=\left[\begin{array}{cccc}
1.2182 & 0 & 0 & 0 \\
-0.21821 & 0 & 0 & \\
0 & 0 & 1 & 1 \\
0 & 0.6 & 0 & 1
\end{array}\right], A_{2}=\left[\begin{array}{cccc}
1.4364 & 0 & 0 & 0 \\
-0.4364 & 1 & 0 & 0 \\
0 & 0 & 1 & 1 \\
0 & 1.2 & 0 & 1
\end{array}\right] \\
B_{1}=\left[\begin{array}{c}
-0.4286 \\
0 \\
0 \\
0
\end{array}\right], B_{2}=\left[\begin{array}{c}
0.871 \\
0 \\
0
\end{array}\right], D_{1}=\left[\begin{array}{c}
0 \\
-1.2 \\
0
\end{array}\right], D_{2}=\left[\begin{array}{c}
0 \\
0 \\
-2.4 \\
0
\end{array}\right], C=\left[\begin{array}{llll}
0 & 1 & 0 & 0 \\
0 & 0 & 1 & 0 \\
0 & 0 & 0 & 1
\end{array}\right]
\end{gathered}
$$

The weighting functions $\rho_{i}(\theta(k))$ are

$$
\rho_{1}(\theta(k))=\frac{\theta(k)-\bar{\theta}}{\underline{\theta}-\bar{\theta}}=\frac{\theta(k)+1.2}{0.6}, \rho_{2}(\theta(k))=\frac{\theta(k)-\underline{\theta}}{\bar{\theta}-\underline{\theta}}=\frac{\theta(k)+0.6}{-0.6}
$$

In this paper, actuator faults are considered. Therefore, it is assumed that $F_{i}=B_{i}, i=1,2$.

\section{Remark 5}

In [20], discrete-time LPV descriptor systems with constant fault distribution matrix are considered.

If the speed is time-varying, the fault distribution matrix in the truck-trailer system is parameterdependent. As a result, the method in [20] cannot be applied in this situation.

By choosing $\gamma_{d}=0.2, \gamma_{f}=7, \gamma_{L}=0.8, \gamma_{N}=1$ and solving the LMIs in (38), we obtain

$$
T=\left[\begin{array}{ccccc}
1 & 2.0719 & -0.0002 & 0.8658 & 0 \\
0 & 0.2442 & 0.0015 & -0.4332 & 0 \\
0 & 0.0006 & 0.0105 & -0.0102 & 0 \\
0 & -0.0003 & -0.0105 & 0.0090 & 0 \\
0 & -0.4532 & -0.0009 & -0.1365 & 1
\end{array}\right], N=\left[\begin{array}{ccc}
-2.0719 & 0.0002 & -0.0002 \\
0.7558 & -0.0015 & 0.0015 \\
-0.0006 & 0.9895 & 0.0105 \\
0.0003 & 0.0105 & 0.9895 \\
0.4532 & 0.0009 & -0.0009
\end{array}\right]
$$




$$
L_{1}=\left[\begin{array}{ccc}
-0.1626 & 0.0001 & 0.4070 \\
0.0767 & -0.0005 & -0.1940 \\
-0.0030 & 0.0053 & -0.0004 \\
0.0040 & -0.0054 & 0.0001 \\
0.0253 & 0.0001 & -0.0695
\end{array}\right], L_{2}=\left[\begin{array}{ccc}
0.2989 & -0.0004 & 0.4248 \\
0.0376 & -0.0004 & -0.1972 \\
-0.0059 & 0.0050 & 0.0000 \\
0.0056 & -0.0051 & -0.0003 \\
-0.1874 & 0.0001 & -0.0753
\end{array}\right]
$$

In the simulation, the time-varying parameter $\theta(k)$ is shown in Fig. 1 and the weighting functions $\rho_{1}(\theta(k)), \rho_{2}(\theta(k))$ are depicted in Fig. 2. It is assumed that the measurements are corrupted by zero-mean Gaussian noises. Specifically, the standard deviations of noises in the measurements of $x_{2}$ and $x_{3}$ are $0.017\left(0.1^{\circ}\right)$ and $0.1 \mathrm{~m}$, respectively. In the following simulation, the initial state is $x(0)=\left[\begin{array}{llll}0.1745 & 0.3491 & 3 & -0.4189\end{array}\right]^{T}$ while the initial estimate is $\hat{\bar{x}}(0)=\left[\begin{array}{lllll}0 & 0 & 0 & 0 & 0\end{array}\right]^{T}$.

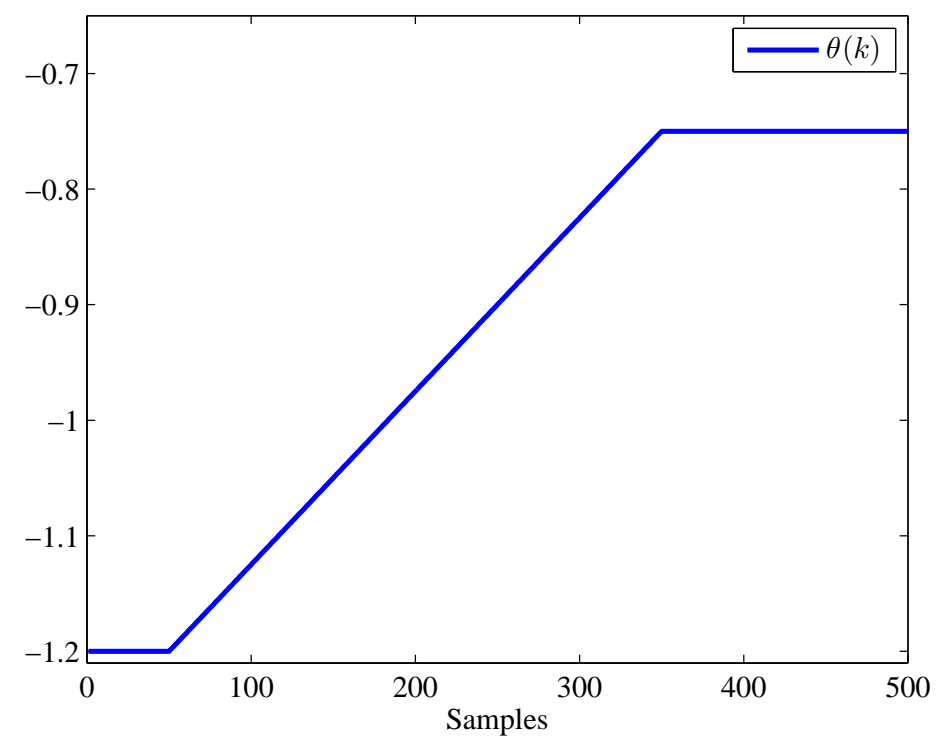

Figure 1. The time-varying parameter $\theta(k)$

First, an abrupt fault is simulated. It is assumed that the abrupt fault is represented by

$$
f(k)=\left\{\begin{array}{cc}
0 & k<200 \\
-1.5 & k \geq 200
\end{array}\right.
$$

The fault estimation result of the robust fault estimation observer is depicted in Fig. 3. Therein, the actual fault is depicted by dash-and-dot line and the fault estimation is represented by the solid 


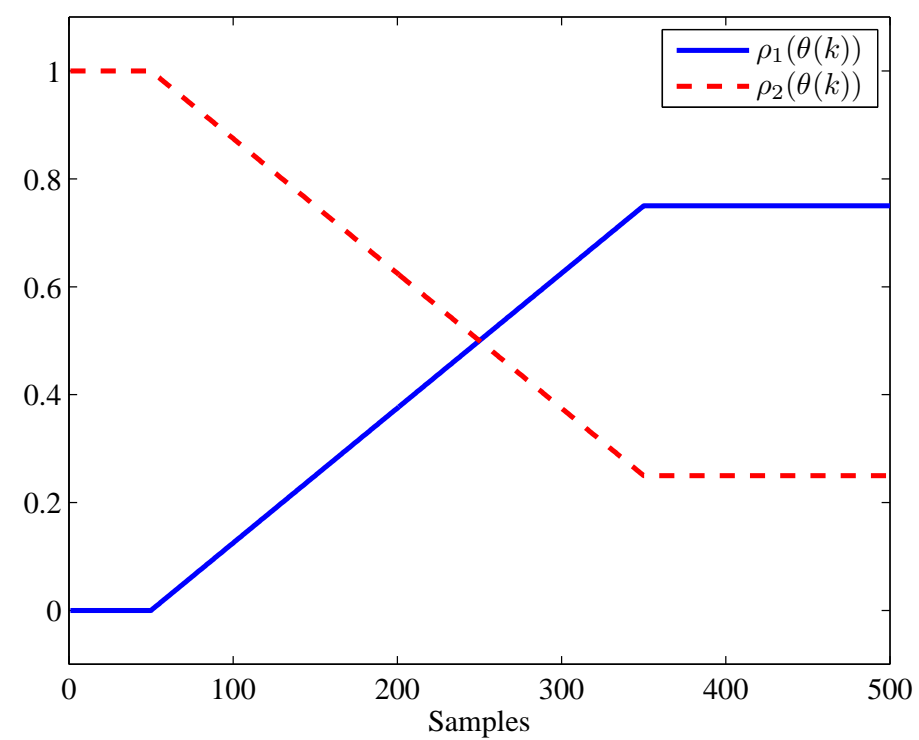

Figure 2. The weighting functions $\rho_{i}(\theta(k)), i=1,2$

one. As shown in Fig. 3, the robust fault estimation observer is insensitive to the model uncertainty and measurement noise. Moreover, although there is initial estimation error, the fault estimate can quickly track the actual fault. This illustrates the fast convergence rate of the fault estimation observer in the face of initial estimation error.

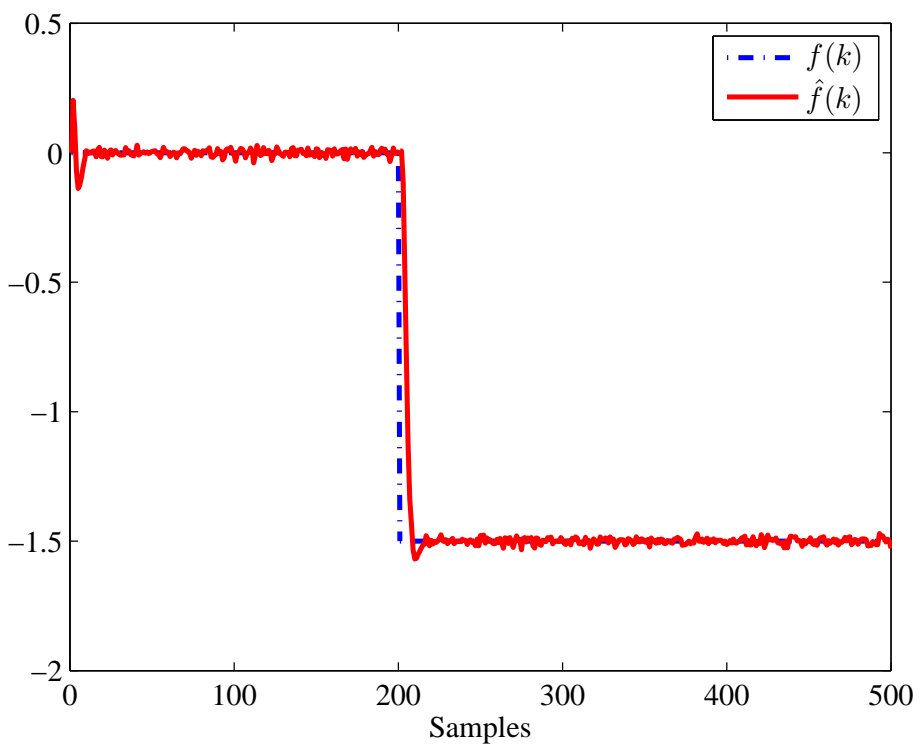

Figure 3. Fault estimation result of the robust fault estimation observer in an abrupt fault scenario 
To illustrate the performance of robust fault estimation observer in dealing with time-varying fault, the following fault is further considered

$$
f(k)=\left\{\begin{array}{cc}
0 & k<100 \\
2 \sin (0.05(k-100)) & k \geq 100
\end{array}\right.
$$

In this situation, the fault estimation result is depicted in Fig. 4. It can be seen from Fig. 4 that there is a delay between the fault $f(k)$ and its estimate. As pointed out in Remark 4, this phenomenon results from the influence of the fault variation.

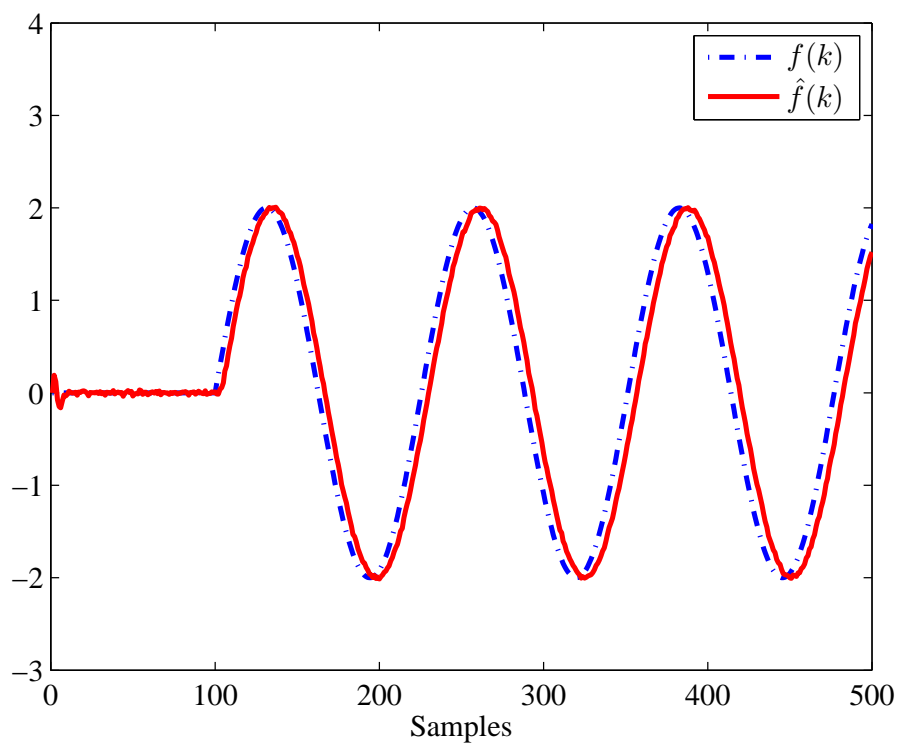

Figure 4. Fault estimation result of the robust fault estimation observer in a time-varying fault scenario

Besides, it is worth mentioning that actuator fault estimation for truck-trailer system has been studied in [28]. However, only a constant speed scenario $(\theta(k)=-1)$ is considered in [28]. In the sequel, this situation is considered so that the method from [28] can be used to compared with our approach. In this situation, the weighting functions $\rho_{1}(\theta(k)), \rho_{2}(\theta(k))$ are depicted in Fig. 5.

Consider the following fault

$$
f(k)=\left\{\begin{array}{cc}
0 & k<100 \\
4\left(1-\mathrm{e}^{-0.05(k-100)}\right) & k \geq 100
\end{array}\right.
$$

The fault estimation results of our method and that of [28] are shown in Fig. 6 and Fig. 7, respectively. In [28], pole assignment is used to ensure the fault estimation convergence speed while 


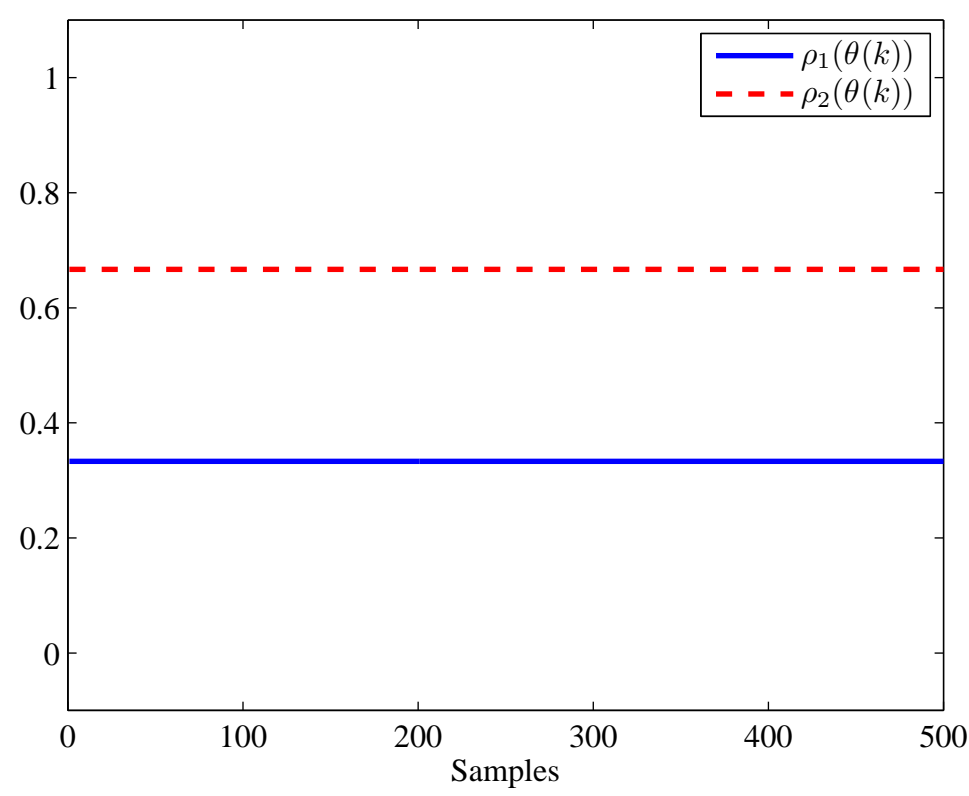

Figure 5. The weighting functions $\rho_{i}(\theta(k)), i=1,2$ when $\theta(k)=1$

our method utilizes $H_{\infty}$ technique to attenuate the effect of fault variation. From Fig.6 and Fig. 7, it can be seen that these two methods have similiar fault convergence speed. Nevertheless, as the measurement noise is not considered in [28], the proposed method is more insensitive to the measurement noise than the one from [28]. Therefore, this simulation result shows the superiority of our method in attenuating measurement noise.

\section{CONCLUSION}

In this paper, the actuator fault estimation problem for discrete-time LPV descriptor systems is considered. First, an augmented system is constructed by considering the fault as an auxiliary state vector. Based on the augmented system, this paper proposes a novel fault estimation observer and presents an LMI-based design method. Considering the unknown disturbance, fault variation, and measurement noise in the practical systems, a robust fault estimation observer design method is proposed. Finally, a truck-trailer model is used to demonstrate the effectiveness and performance of the proposed method. 


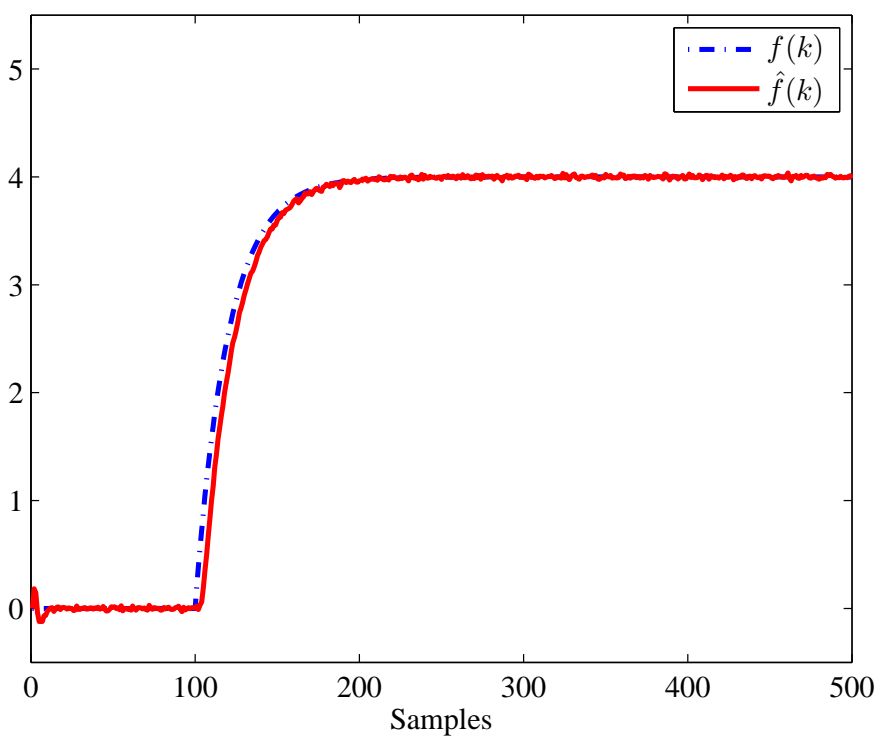

Figure 6. Fault estimation result of the proposed method

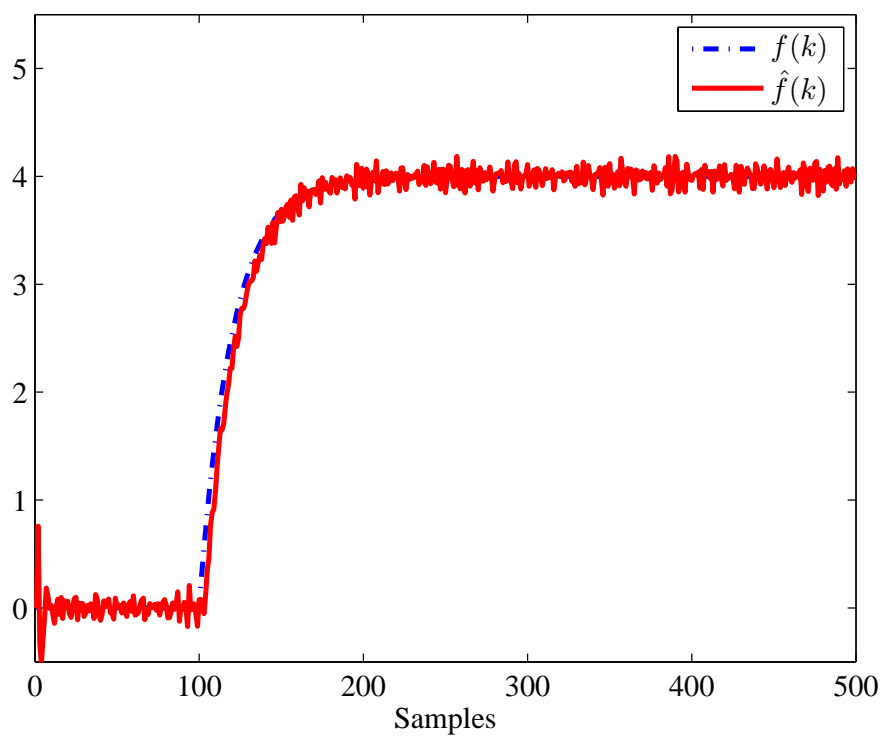

Figure 7. Fault estimation result of the method from [28]

\section{ACKNOWLEDGEMENT}

This work was financially supported by the National Natural Science Foundation of China (Grant No. 61273162). The authors would like to thank the anonymous reviewers and the associate editor for their helpful remarks which improved the quality of this paper. 


\section{REFERENCES}

[1] Chen J, Patton R. 1999. Robust Model-Based Fault Diagnosis for Dynamic Systems. Kluwer Academic Publishers, Boston.

[2] Ding S X. 2008. Model-Based Fault Diagnosis Techniques. Springer, Berlin.

[3] Frank P M. Fault diagnosis in dynamic systems using analytical and knowledge based redundancy-A survey and some new results. Automatica 1990; 26(3): 459-474.

[4] Hwang I, Kim S, Kim Y, Seah C E. A survey of fault detection, isolation, and reconfiguration methods. IEEE Transactions on Control Systems Technology 2010; 18(3): 636-653.

[5] Alcorta-García E, Frank P M. Deterministic nonlinear observer based approaches to fault diagnosis: A survey. Control Engineering Practice 1997; 5(5): 663-670.

[6] Bokor J, Szabó Z. Fault detection and isolation in nonlinear systems. Annual Reviews in Control 2009; 33(2): 113-123.

[7] Mohammadpour J, Scherer C M (eds). 2012. Control of Linear Parameter-Varying Systems with Applications. Springer, New York.

[8] Tóth R. 2010. Modeling and Identification of Linear Parameter-Varying Systems. Springer, Berlin.

[9] Alwi H, Edwards C, Marcos A. Fault reconstruction using a LPV sliding mode observer for a class of LPV systems. Journal of the Franklin Institute 2012; 349(2): 510-530.

[10] Kulcsár B, Verhaegen M. Robust inversion based fault estimation for discrete-time LPV systems. IEEE Transactions on Automatic Control 2012; 57(6): 1581-1586.

[11] Rodrigues M, Sahnoun M, Theilliol D, Ponsart J C. Sensor fault detection and isolation filter for polytopic LPV systems: A winding machine application. Journal of Process Control 2013; 23(6): 805816.

[12] Dai L. 1989. Singular Control Systems. Springer, Belin.

[13] Duan G R. 2010. Analysis and Design of Descriptor Linear Systems. Springer, New York.

[14] Vemuri A T, Polycarpou M M, Ciric A R. Fault diagnosis of differential-algebraic systems. IEEE Transactions on Systems, Man, and Cybernetics 2001; 31(2): 143-152. 
[15] Duan G R, Howe D, Patton R J. Robust fault detection in descriptor linear systems via generalized unknown input observers. International Journal of Systems Science 2002; 33(5): 369-377.

[16] Yeu T K, Kim H S, Kawaji S. Fault detection, isolation and reconstruction for descriptor systems. Asian Journal of Control 2005; 7(4): 356-367.

[17] Lin J, Fei S, Gao Z, Ding J. Fault detection for discrete-time switched singular time-delay systems: An average dwell time approach. International Journal of Adaptive Control and Signal Processing 2013; 27(7): 582-609.

[18] Boulkroune B, Halabi S, Zemouche A. $H_{-} / H_{\infty}$ fault detection filter for a class of nonlinear descriptor systems. International Journal of Control 2013; 86(2): 253-262.

[19] Hamdi H, Rodrigues M, Mechmeche C, Theilliol D, Braiek N B. Fault detection and isolation in linear parameter varying descriptor systems via proportional integral observer. International Journal of Adaptive Control and Signal Processing 2012; 26(3): 224-240.

[20] Astorga-Zaragoza C M, Theilliol D, Ponsart J C, Rodrigues M. Fault diagnosis for a class of descriptor linear parameter-varying systems. International Journal of Adaptive Control and Signal Processing 2012; 26(3): 208-223.

[21] Zhang Y, Jiang J. Bibliographical review on reconfigurable fault-tolerant control systems. Annual Reviews in Control 2008; 32(2): 229-252.

[22] Chadli M, Abdo A, Ding S X. $H_{-} / H_{\infty}$ fault detection filter design for discrete-time Takagi-Sugeno fuzzy system. Automatica 2013; 49(7): 1996-2005.

[23] Zhang K, Jiang B, Shi P. Observer-based integrated robust fault estimation and accommodation design for discrete-time systems. International Journal of Control 2010; 83(6): 1167-1181.

[24] Ben-Israel A, Greville T. 2003. Generalized Inverses: Theory and Applications (2nd edn). Springer, New York.

[25] Boyd S, Ghaoui L, Feron E, Balakrishnan V. 1994. Linear Matrix Inequalities in System and Control Theory Society for Industrial and Applied Mathematics (SIAM), Philadelphia. Addison-Wesley.

[26] Marx B, Koenig D, Ragot J. Design of observers for Takagi-Sugeno descriptor systems with unknown inputs and application to fault diagnosis. IET Control Theory \& Applications 2007; 1(5): 1487-1495. 
[27] Tanaka K, Sano M. A robust stabilization problem of fuzzy control systems and its application to backing up control of a truck-trailer. IEEE Transactions on Fuzzy Systems 1994; 2(2): 119-134.

[28] Zhang K, Jiang B, Shi P. Fault estimation observer design for discrete-time Takagi-Sugeno fuzzy systems based on piecewise Lyapunov functions. IEEE Transactions on Fuzzy Systems 2012; 20(1): 192-200. 